\title{
The Size of the Largest Part of Random Weighted Partitions of Large Integers
}

\author{
Ljuben Mutafchiev \\ American University in Bulgaria, 2700 Blagoevgrad, Bulgaria \\ and Institute of Mathematics and Informatics of the \\ Bulgarian Academy of Sciences \\ ljuben@aubg.bg
}

\begin{abstract}
We consider partitions of the positive integer $n$ whose parts satisfy the following condition: for a given sequence of non-negative numbers $\left\{b_{k}\right\}_{k \geq 1}$, a part of size $k$ appears in exactly $b_{k}$ possible types. Assuming that a weighted partition is selected uniformly at random from the set of all such partitions, we study the asymptotic behavior of the largest part $X_{n}$. Let $D(s)=\sum_{k=1}^{\infty} b_{k} k^{-s}, s=\sigma+i y$, be the Dirichlet generating series of the weights $b_{k}$. Under certain fairly general assumptions, Meinardus (1954) has obtained the asymptotic of the total number of such partitions as $n \rightarrow \infty$. Using the Meinardus scheme of conditions, we prove that $X_{n}$, appropriately normalized, converges weakly to a random variable having Gumbel distribution (i.e. its distribution function equals $e^{-e^{-t}},-\infty<$ $t<\infty)$. This limit theorem extends some known results on particular types of partitions and on the Bose-Einstein model of ideal gas.
\end{abstract}

Mathematics Subject classifications: 05A17, 60C05, 60F05

\section{Introduction and Statement of the Result}

A weighted partition of the positive integer $n$ is a multiset of size $n$ whose decomposition into a union of disjoint components (parts) satisfies the following condition: for a given sequence of non-negative numbers $\left\{b_{k}\right\}_{k \geq 1}$, a part of size $k$ appears in exactly one of $b_{k}$ possible types. For more details on properties of multisets, we refer the reader e.g. to [3]. Weighted partitions are also associated with the generalized Bose-Einstein model of ideal gas, where $n(=E)$ is interpreted as the total energy of the system of particles. The weights $b_{k}, k \geq 1$, are viewed as counts of the distinct positions of the particles in the state space, where a particle in a given position has (rescaled) energy $k$ (for more details on the relationship between combinatorial partitions and various models of ideal 
gas, see [21]). From combinatorial point of view, it is fairly natural to assume that $b_{k}, k \geq 1$, are integers (see e.g. the "money changing problem" discussed in detail in [24; Sect. 3.15]). On the other hand, it turns out that this requirement is not necessary for the analytical approach used in this paper. That is why, we assume that $b_{k}, k \geq 1$, are real non-negative numbers.

For a given sequence $b=\left\{b_{k}, k \geq 1\right\}$, let $\mathcal{P}_{b}(n)$ be the set of all weighted partitions of the positive integer $n$ and let $p_{b}(n)=\left|\mathcal{P}_{b}(n)\right|$ be its cardinality. It is known that the generating function $f_{b}(x)$ of the numbers $p_{b}(n)$ is of Euler's type, namely,

$$
f_{b}(x)=1+\sum_{n=1}^{\infty} p_{b}(n) x^{n}=\prod_{k=1}^{\infty}\left(1-x^{k}\right)^{-b_{k}}, \quad|x|<1
$$

(see [24; Sect. 3.14]). We introduce the uniform probability measure $\mathbb{P}=\mathbb{P}_{n, b}$ on the set of weighted partitions of $n$ assuming that the probability $1 / p_{b}(n)$ is assigned to each $n$-partition with weight sequence $b$. In this paper we focus on the size of the largest part $X_{n}$ of a random weighted partition of $n$. With respect to the probability measure $\mathbb{P}, X_{n}$ becomes a random variable, defined on the set $\mathcal{P}_{b}(n)$. It is also well-known that

$$
f_{m, b}(x)=1+\sum_{n=1}^{\infty} p_{b}(n) \mathbb{P}\left(X_{n} \leq m\right) x^{n}=\prod_{k=1}^{m}\left(1-x^{k}\right)^{-b_{k}}, \quad m \geq 1
$$

(see [24; Sect. 3.15]).

The asymptotic behavior of the combinatorial numbers $p_{b}(n)$ (the Taylor coefficients in (1.1) ) will play important role in our further analysis. A fairly general scheme of assumptions on the parametric sequence $b$ was proposed by Meinardus [13] (see also [2; Chap. 6]), who found an asymptotic expansion for the numbers $p_{b}(n)$ as $n \rightarrow \infty$. The same asymptotic was also obtained in [10], where one of the Meinardus conditions was weakened. Meinardus' approach is based on considering two generating series:

$$
D(s)=\sum_{k=1}^{\infty} b_{k} k^{-s}, \quad s=\sigma+i y,
$$

and

$$
G(z)=\sum_{k=1}^{\infty} b_{k} z^{k}, \quad|z| \leq 1
$$

Below we give the Meinardus scheme of conditions. Throughout the paper by $\Re(z)$ and $\Im(z)$ we denote the real and imaginary part of the complex number $z$, respectively.

$\left(M_{1}\right)$ The Dirichlet series (1.3) converges in the half-plane $\sigma>\rho>0$ and there is a constant $C_{0} \in(0,1)$, such that the function $D(s)$ has an analytic continuation to the half-plane $\left\{s: \sigma \geq-C_{0}\right\}$ on which it is analytic except for the simple pole at $s=\rho$ with residue $A>0$. 
$\left(M_{2}\right)$ There exists a constant $C_{1}>0$ such that

$$
D(s)=O\left(|y|^{C_{1}}\right), \quad|y| \rightarrow \infty,
$$

uniformly for $\sigma \geq-C_{0}$.

$\left(M_{3}\right)$ There are constants $\epsilon>0$ and $C_{2}\left(=C_{2}(\epsilon)>0\right)$, such that the function $g(\tau)=G\left(e^{-\tau}\right), \tau=\alpha+2 \pi i u, u$ real and $\alpha>0$ (see (1.4)) satisfies

$$
\Re(g(\tau))-g(\alpha) \leq-C_{2} \alpha^{-\epsilon}, \quad|\arg (\tau)|>\pi / 4, \quad 0 \neq|u| \leq 1 / 2,
$$

for enough small values of $\alpha$.

The first assumption (M1) specifies the domain, say $\mathcal{H}$, in which $D(s)$ has an analytic continuation. The second is related to the asymptotic behavior of $D(s)$, whenever $|\Im(s)| \rightarrow \infty$. Functions, which are bounded by $O\left(|\Im(s)|^{q}\right), 0<q<\infty$, in certain domain, as $|\Im(s)| \rightarrow \infty$, are called functions of finite order. It is known that the sum of the Dirichlet series in (1.3) satisfies the finite order property in a closed half-plane contained in the half-plane of convergence $\sigma>\rho$ (see e.g. [20; Sect. 9.3.3]). The Meinardus second condition requires that the same holds for the analytic continuation of $D(s)$ in the whole domain $\mathcal{H}$. Finally, the Meinardus third condition implies a bound on $\Re\left(G\left(e^{-\tau}\right)\right.$ ) (see (1.4) ) for certain specific complex values of $\tau$. In some cases its verification is technically complicated. Since

$$
\Re(g(\tau))-g(\alpha)=-2 \sum_{k=1}^{\infty} b_{k} e^{-k \alpha} \sin ^{2}(\pi k u)
$$

and the inequality $|\arg (\tau)|>\pi / 4$ implies that $\tan (|\arg (\tau)|)=2 \pi|u| / \alpha>1$, condition $\left(M_{3}\right)$ can be also reformulated as follows:

$$
S_{n}:=\sum_{k=1}^{\infty} b_{k} e^{-k \alpha} \sin ^{2}(\pi k u) \geq C_{2} \alpha^{-\epsilon}, \quad 0<\frac{\alpha}{2 \pi}<|u| \leq 1 / 2,
$$

for small enough $\alpha$ and some constants $C_{2}, \epsilon>0\left(C_{2}=C_{2}(\epsilon)\right)$ [10; p.310].

Moreover, Granovsky et al. [10; Lemma 1] proved that this inequality holds for any sequence $b_{k}, k \geq 1$, satisfying the inequality $b_{k} \geq C k^{\nu-1}, k \geq k_{0}$, for some $k_{0} \geq 1$ and $C, \nu>0$. We notice that if

$$
b_{k}=C k^{\nu-1}, \quad k \geq 1,
$$

then $D(s)=C \zeta(s-\nu+1)$, where $\zeta$ denotes the Riemann zeta function. Therefore, $D(s)$ has a single pole at $s=\nu$ with residue $C>0$ and a meromorphic analytic continuation to the whole complex plane [23; Sect. 13.13]. These facts show that conditions $\left(M_{1}\right)-\left(M_{3}\right)$ are satisfied by the weights (1.7) with $\rho=\nu$ and $A=C$.

Throughout the paper we assume that conditions $\left(M_{1}\right)-\left(M_{3}\right)$ are satisfied. Our aim is to determine asymptotically, as $n \rightarrow \infty$, the distribution of the maximal part size $X_{n}$. Recalling (1.2), we also point out that our results may be 
interpreted in terms of the asymptotic of the combinatorial counts of partitions whose part sizes are $\leq m$, where the range of the values of $m$ is specified by the weak convergence of the random variable $X_{n}$ to a non-degenerate random variable. In the brief review given below we summarize some known results on the limiting behavior of the random variable $X_{n}$.

Consider first the classical case of linear integer partitions, where the weights satisfy $b_{k}=1, k \geq 1$. This kind of partitions were broadly studied by many authors in many respects. Their graphical representations by Ferrers diagrams show that their total number of parts and their maximal part size $X_{n}$ are identically distributed for all $n$ (see [2; Sect. 1.3]). Erdös and Lehner [6] were apparently the first who applied a probabilistic approach to the study of integer partitions. As a matter of fact, they found an appropriate normalization for $X_{n}$ in this case and showed that $\pi X_{n} /(6 n)^{1 / 2}-\log \left((6 n)^{1 / 2} / \pi\right)$ converges weakly, as $n \rightarrow \infty$, to a random variable having the extreme value (Gumbel) distribution. The local version of their theorem was derived later by Auluck et al. [4]. Fristedt [9] studied linear integer partitions using a transfer method to functionals of independent and geometrically distributed random variables. Among other results, he obtained the limiting distribution of the $k$ th largest part size whenever $k$ is fixed. Finally, we notice that among weighted integer partitions only the linear ones possess the property that the number of parts and the maximum part size are identically distributed. The limiting distribution of the number of parts in the general case of random weighted partitions under the Meinardus scheme of conditions is studied in [15]. It turns out that the limiting distribution laws depend on particular ranges in which the parameter $\rho$ varies (see condition $\left(M_{1}\right)$ ).

Another important particular case of weighted partitions arises whenever $b_{k}=k, k \geq 1$. It turns out that in this case the generating function $f_{b}(x)$ (see (1.1) ) enumerates the plane partitions. A plane partition of $n \geq 1$ is a matrix of non-negative integers arranged in non-increasing order from left to right and from top to bottom, so that the double sum of its elements equals $n$. Together with the largest part size $X_{n}$, consider also the counts of the nonzero rows and columns of the matrix of a plane partition. It turns out that these three quantities measure the sizes of the corresponding solid diagram of a plane partition in the $3 D$ space. (The solid diagram is a heap of $n$ unit cubes placed in the first octant of a coordinate system in a $3 D$ space whose columns composed by stacked cubes have non-increasing heights along the $x$ - and $y$ axis; the height of this heap along the $z$-axis is just $X_{n}$, the largest part size.) Similarly to Ferrers diagrams for linear integer partitions, the three sizes of this heap appear to be identically distributed for all $n \geq 1$ (for more details, see $[19 ;$ p. 371]). Their joint limiting distribution was found in [17]. The marginal limiting distributions (including the limiting distribution of $X_{n}$ ) were obtained in [14]. For more details on various properties of plane partitions and their applications to combinatorics and analysis of algorithms, we refer the reader to [2; Chap. 11], [16; Chap. 11] and [19; Chap. 7].

Our study is also closely related to some recent results on the maximal particle energy in the Bose-Einstein model of ideal gas. The general setting 
and the probabilistic frame of problems from statistical mechanics and their relationship with enumerative combinatorics were given by Vershik [21]. In the context of the infinite product formula (1.1), he studied the Bose-Einstein model by a family of probability measures $\mu_{v}, v \in(0,1)$, defined on the set of all $b$-weighted partitions $\mathcal{P}_{b}=\cup_{n \geq 0} \mathcal{P}_{b}(n)$. So, for a partition $\lambda=\left(\lambda_{1}, \ldots, \lambda_{l}\right) \in$ $\mathcal{P}, \lambda_{1} \geq \ldots \geq \lambda_{l}>0$, let $r_{k}(\lambda)=\left\{j: \lambda_{j}=k\right\}$ denote the number of parts of $\lambda$ that are equal to $k \geq 1$. Then $\mu_{v}$ is defined by

$$
\mu_{v}\left(\left\{\lambda \in \mathcal{P}: r_{k}(\lambda)=j\right\}\right)=\left(\begin{array}{c}
b_{k}+j-1 \\
j
\end{array}\right) v^{k j}\left(1-v^{k}\right)^{b_{k}}, \quad 0<v<1 .
$$

The key feature in the study of this kind of measures is the fact that a kind of a conditional probability measure on $\mathcal{P}_{b}(n)$ turns out to be independent of $v$ for all $n$ and coincides with the uniform probability measure $\mathbb{P}=\mathbb{P}_{n, b}$ (for more details, see [21]). In [22] Vershik and Yakubovich studied the limiting distribution of the maximal particle energy $X(\lambda)$, or, which is the same, the largest part size $X(\lambda), \lambda \in \mathcal{P}$, with respect to the measure $\mu_{v}$, as $v \rightarrow 1^{-}$. In particular, under the assumption that the weight sequence $b$ satisfies (1.7), they proved that

$$
\begin{aligned}
& \lim _{v \rightarrow 1^{-}} \mu_{v}(\{\lambda \in \mathcal{P}:(1-v) X(\lambda)-\nu|\log (1-v)| \\
& -(\nu-1) \log |\log (1-v)|-(\nu-1) \log \nu-\log C \leq t\}) \\
& =e^{-e^{-t}}, \quad-\infty<t<\infty .
\end{aligned}
$$

As it was mentioned before, the weight sequence (1.7) satisfies conditions $\left(M_{1}\right)$ $\left(M_{3}\right)$. Vershik and Yakubovich [22] studied also a more realistic model of quantum ideal gas in a $N$-dimensional space, for which the weights satisfy $\sum_{j=1}^{k} b_{j}=K_{N} k^{N / 2}+O\left(k^{\kappa_{N}}\right)$, as $k \rightarrow \infty\left(K_{N}\right.$ and $\kappa_{N}<N / 2$ are computable constants).

The main result of this paper is obtained in terms of the uniform probability measure $\mathbb{P}=\mathbb{P}_{n, b}$ on the set $\mathcal{P}_{b}(n)$. Before stating it, for the sake of brevity, we introduce the following notation:

$$
a(n)=a(n ; \rho, A)=\left(\frac{A \Gamma(\rho+1) \zeta(\rho+1)}{n}\right)^{\frac{1}{\rho+1}}, \quad n \geq 1,
$$

where the constants $\rho$ and $A$ are defined by condition $(M 1)$.

Theorem 1 If the weight sequence b, satisfies conditions $(M 1)-(M 3)$, then, for all real $t$, the limiting distribution of the largest part size $X_{n}$ is given by

$$
\begin{aligned}
& \lim _{n \rightarrow \infty} \mathbb{P}\left(a(n) X_{n}+\rho \log a(n)-(\rho-1) \log |\log a(n)|-(\rho-1) \log \rho-\log A \leq t\right) \\
& =e^{-e^{-t}} .
\end{aligned}
$$

Remark. One can easily compare (1.10) with (1.8) setting in the latter one $v=1-a(n), \nu=\rho$ and $C=A$ and observe the coinciding normalizations. We 
also notice that the limiting results for linear and plane partitions (see $[6,14]$ ) follow from (1.10) with $A=1$ and $\rho=1$ and 2, respectively.

The method of our proof combines Hayman's theorem for estimating coefficients of admissible power series [11] (see also [7; Sect. VIII.5]), a generalization of Perron formula, which yields the expression for partial sums of a Dirichlet series by a complex integral of the inverse Mellin transform applied to the Dirichlet series itself (see Thm 3.1 from the Supplement of [18]) and some Mellin transform computations.

We organize our paper as follows. Section 2 includes some auxiliary facts that we need further. Some proofs are omitted since they are given in $[10,13]$. In Section 3 we present the proof of Theorem 1. The Appendix contains some technical details related to the application of the generalized Perron formula [18].

\section{Preliminary Results}

We start with a lemma establishing an asymptotic estimate for infinite product representation (1.1) of the generating function $f_{b}(x)$. It has been proved by Meinardus [13] (see also [2; Lemma 6.1]).

Lemma 1 Suppose that sequence $b$ is such that the associated Dirichlet series (1.3) satisfies conditions $\left(M_{1}\right)$ and $\left(M_{2}\right)$. If $\tau=\alpha+i \theta$, then

$$
f_{b}\left(e^{-\tau}\right)=\exp \left(A \Gamma(\rho) \zeta(\rho+1) \tau^{-\rho}-D(0) \log \tau+D^{\prime}(0)+O\left(\alpha^{C_{0}}\right)\right)
$$

as $\alpha \rightarrow 0^{+}$uniformly for $|\theta| \leq \pi$ and $|\arg \tau| \leq \pi / 4$.

Our first goal is to show that Meinardus' conditions $\left(M_{1}\right)-\left(M_{3}\right)$ imply that the generating function $f_{b}(x)$ possesses the Hayman admissibility properties [11] (see also [7; Sect. VIII.5]) in the unit disc. For $0<r<1$, we introduce the functions:

$$
\begin{gathered}
F_{b}(r)=\log f_{b}(r)=-\sum_{k=1}^{\infty} b_{k} \log \left(1-r^{k}\right), \\
\mathcal{A}_{b}(r)=r F_{b}^{\prime}(r)=r \frac{f_{b}^{\prime}(r)}{f_{b}(r)} \\
\mathcal{B}_{b}(r)=r^{2} F_{b}^{\prime \prime}(r)+r F_{b}^{\prime}(r)=r \frac{f_{b}^{\prime}(r)}{f_{b}(r)}+r^{2} \frac{f_{b}^{\prime \prime}(r)}{f_{b}(r)}-r^{2}\left(\frac{f_{b}^{\prime}(r)}{f_{b}(r)}\right)^{2} .
\end{gathered}
$$

Furthermore, setting in (2.1)-(2.3) $r=e^{-\alpha}$, we shall obtain their asymptotic expansions as $\alpha \rightarrow 0^{+}$. For the sake of convenience, we also set

$$
h=h(\rho, A)=A \Gamma(\rho+1) \zeta(\rho+1) .
$$

The proof of the next lemma is contained in [10; Lemma 2]. 
Lemma 2 Conditions $\left(M_{1}\right)$ and $\left(M_{2}\right)$ imply the following asymptotic expansions:

$$
\begin{gathered}
\mathcal{A}_{b}\left(e^{-\alpha}\right)=h \alpha^{-\rho-1}+D(0) \alpha^{-1}+D^{\prime}(0)+O\left(\alpha^{C_{0}-1}\right), \\
\mathcal{B}_{b}\left(e^{-\alpha}\right)=\frac{d}{d \alpha}\left(-\mathcal{A}_{b}\left(e^{-\alpha}\right)\right)=h(\rho+1) \alpha^{-\rho-2}+D(0) \alpha^{-2}+O\left(\alpha^{C_{0}-2}\right), \\
F_{b}^{\prime \prime \prime}\left(e^{-\alpha}\right)=O\left(\alpha^{-\rho-3}\right),
\end{gathered}
$$

as $\alpha \rightarrow 0^{+}$, where $F_{b}, \mathcal{A}_{b}$ and $\mathcal{B}_{b}$ are defined by (2.1)-(2.3), respectively. Moreover, from (2.5) it follows that the equation

$$
\mathcal{A}_{b}\left(e^{-\alpha}\right)=n, \quad n \geq 1,
$$

has a unique solution $\alpha=\alpha_{n}$, such that $\alpha_{n} \rightarrow 0$ as $n \rightarrow \infty$. An asymptotic expansion of this solution, as $n \rightarrow \infty$, is given by

$$
\alpha_{n}=a(n)+\frac{D(0)}{(\rho+1) n}+O\left(n^{-1-\beta}\right)
$$

where $\beta=\min \left(\frac{C_{0}}{\rho+1}, \frac{\rho}{\rho+1}\right)$ and $a(n)$ are the normalizing constants given by (1.9).

We notice that (2.5), (2.6) and (2.9) imply that

$$
\mathcal{A}_{b}\left(e^{-\alpha_{n}}\right) \rightarrow \infty, \quad \mathcal{B}_{b}\left(e^{-\alpha_{n}}\right) \rightarrow \infty, \quad n \rightarrow \infty,
$$

that is, Hayman "capture" condition [7; p. 565] is satisfied with $r=r_{n}=e^{-\alpha_{n}}$. Our next step is to establish Hayman "locality" condition, which implies the asymptotic behavior of $f_{b}(x)$ in a suitable neighborhood of $x=1$.

Lemma 3 Suppose that the weight sequence $b$ satisfies conditions $\left(M_{1}\right)$ and $\left(M_{2}\right)$ and $\alpha_{n}$ is the solution of (2.8) given by (2.9). Let

$$
\delta_{n}=\alpha_{n}^{1+\rho / 3} / \omega(n), \quad n \geq 1,
$$

where $\omega(n) \rightarrow \infty$ as $n \rightarrow \infty$ arbitrarily slowly. Then

$$
e^{-i \theta n} \frac{f_{b}\left(e^{-\alpha_{n}+i \theta}\right)}{f_{b}\left(e^{-\alpha_{n}}\right)}=e^{-\theta^{2} \mathcal{B}_{b}\left(e^{-\alpha_{n}}\right) / 2}\left(1+O\left(1 / \omega^{3}(n)\right)\right.
$$

uniformly for $|\theta| \leq \delta_{n}$.

Proof. Applying Lemma 1, we observe that

$$
\begin{aligned}
& e^{-i \theta n} \frac{f_{b}\left(e^{-\alpha_{n}+i \theta}\right)}{f_{b}\left(e^{-\alpha_{n}}\right)} \\
& =\exp \left(\frac{h}{\rho}\left(\left(\alpha_{n}-i \theta\right)^{-\rho}-\alpha_{n}^{-\rho}\right)-D(0) \log \left(1-\frac{i \theta}{\alpha_{n}}\right)-i \theta n+O\left(\alpha_{n}^{C_{0}}\right)\right),
\end{aligned}
$$


where $h$ is given by (2.4). Expanding $\left(\alpha_{n}-i \theta\right)^{-\rho}$ and $\log \left(1-i \theta / \alpha_{n}\right)$ by Taylor formula and using (2.5), (2.6) and (2.8), we obtain

$$
\begin{aligned}
& \frac{h}{\rho}\left(\left(\alpha_{n}-i \theta\right)^{-\rho}-\alpha_{n}^{-\rho}\right)-D(0) \log \left(1-\frac{i \theta}{\alpha_{n}}\right)-i \theta n \\
& =i \theta\left(h \alpha_{n}^{-\rho-1}+D(0) \alpha_{n}^{-1}+D^{\prime}(0)-n\right)-\frac{\theta^{2}}{2} h(\rho+1) \alpha_{n}^{-\rho-2}-\frac{D(0) \theta^{2}}{2 \alpha_{n}^{2}} \\
& -i \theta D^{\prime}(0)+O\left(|\theta|^{3} \alpha_{n}^{-3-\rho}\right)=i \theta\left(\mathcal{A}_{b}\left(e^{-\alpha_{n}}\right)-n+O\left(\alpha_{n}^{C_{0}-1}\right)\right) \\
& -\frac{\theta^{2}}{2}\left(\mathcal{B}_{b}\left(e^{-\alpha_{n}}\right)+O\left(\alpha_{n}^{C_{0}-2}\right)\right)+O(|\theta|)+O\left(|\theta|^{3} \alpha_{n}^{-3-\rho}\right) \\
& =-\frac{\theta^{2}}{2} \mathcal{B}_{b}\left(e^{-\alpha_{n}}\right)+O\left(\delta_{n} \alpha_{n}^{C_{0}-1}\right)+O\left(\delta_{n}^{2} \alpha_{n}^{C_{0}-2}\right)+O\left(\delta_{n}\right)+O\left(\delta_{n}^{3} \alpha_{n}^{-3-\rho}\right) .
\end{aligned}
$$

Substituting this into (2.13) and taking into account (2.11), we obtain (2.12).

To study the behavior of $f_{b}\left(e^{-\alpha_{n}+i \theta}\right)$ outside the range $-\delta_{n}<\theta<\delta_{n}$ we need the Wiener-Ikehara Tauberian theorem on Dirichlet series. It tells us how condition $\left(M_{1}\right)$ implies an asymptotic estimate for the partial sums $\sum_{k=1}^{n} b_{k}$.

Wiener-Ikehara Theorem. (See [12; Thm. 2.2, p. 122].) Suppose that the Dirichlet series $\tilde{D}(s)=\sum_{k=1}^{\infty} c_{k} k^{-s}$ is such that the function $\tilde{D}(s)-\frac{c}{s-1}$ has an analytic continuation to the closed half-plane $\Re(s) \geq 1$. Then

$$
\sum_{k=1}^{n} c_{k} \sim c n, \quad n \rightarrow \infty
$$

We also denote by $\{\gamma\}$ the fractional part of the real number $\gamma$, and by $\|\gamma\|$ the distance from $\gamma$ to the nearest integer, so that

$$
\|\gamma\|=\left\{\begin{array}{lll}
\{\gamma\} & \text { if } & \{\gamma\} \leq 1 / 2 \\
1-\{\gamma\} & \text { if } & \{\gamma\}>1 / 2
\end{array}\right.
$$

It is not difficult to show that

$$
\sin ^{2}(\pi \gamma) \geq 4\|\gamma\|^{2}
$$

(see $[8 ;$ p. 272]). Now, we are ready to prove that Hayman last ("decay") condition $[7 ;$ p. 565$]$ is also valid.

Lemma 4 Suppose that $f_{b}(x)$ satisfies conditions $\left(M_{1}\right)-\left(M_{3}\right)$. Then, for sufficiently large $n$,

$$
\left|f_{b}\left(e^{-\alpha_{n}+i \theta}\right)\right| \leq f_{b}\left(e^{-\alpha_{n}}\right) e^{-C_{3} \alpha_{n}^{-\epsilon_{1}}}
$$

uniformly for $\delta_{n} \leq|\theta|<\pi$, where $C_{3}$ and $\epsilon_{1}$ are positive constants.

Proof. First, we notice that

$$
\frac{\left|f_{b}\left(e^{-\alpha_{n}+i \theta}\right)\right|}{f_{b}\left(e^{-\alpha_{n}}\right)}=\exp \left(\Re\left(\log f_{b}\left(e^{-\alpha_{n}+i \theta}\right)\right)-\log f_{b}\left(e^{-\alpha_{n}}\right)\right) .
$$


Then, setting $\theta=2 \pi u$, for $\alpha_{n} / 2 \pi \leq|u|=|\theta| / 2 \pi<1 / 2$, we almost repeat the argument from [10; p. 324]:

$$
\begin{aligned}
& \Re\left(\log f_{b}\left(e^{-\alpha_{n}+i \theta}\right)\right)-\log f_{b}\left(e^{-\alpha_{n}}\right) \\
& =\Re\left(-\sum_{k=1}^{\infty} b_{k} \log \left(\frac{1-e^{-k \alpha_{n}+2 \pi i u k}}{1-e^{-k \alpha_{n}}}\right)\right) \\
& =-\frac{1}{2} \sum_{k=1}^{\infty} b_{k} \log \left(\frac{1-2 e^{-k \alpha_{n}} \cos (2 \pi u k)+e^{-2 \alpha_{n} k}}{\left(1-e^{-\alpha_{n} k}\right)^{2}}\right) \\
& =-\frac{1}{2} \sum_{k=1}^{\infty} b_{k} \log \left(1+\frac{4 e^{-\alpha_{n} k} \sin ^{2}(\pi u k)}{\left(1-e^{-\alpha_{n} k}\right)^{2}}\right) \\
& \leq-\frac{1}{2} \sum_{k=1}^{\infty} b_{k} \log \left(1+4 e^{-\alpha_{n} k} \sin ^{2}(\pi u k)\right) \\
& \leq-\frac{\log 5}{2} \sum_{k=1}^{\infty} b_{k} e^{-\alpha_{n} k} \sin ^{2}(\pi u k) \\
& =-\frac{\log 5}{2} S_{n} \leq-\frac{\log 5}{2} C_{2} \alpha_{n}^{-\epsilon},
\end{aligned}
$$

where the last two inequalities follow from the fact that $\log (1+y) \geq\left(\frac{\log 5}{4}\right) y$ $(0 \leq y \leq 4)$ and (1.6), respectively. Thus, the required inequality is proved for $\alpha_{n} \leq|\theta|<\pi$. It remains to consider the interval $\delta_{n} \leq|\theta|<\alpha_{n}$. (1.5) implies that we have to find now a lower bound for the sum $S_{n}$ in (1.6) if $\delta_{n} / 2 \pi \leq|u|<\alpha_{n} / 2 \pi$ (i.e. if $\delta_{n} \leq|\theta|<\alpha_{n}$ ). We shall apply Wiener-Ikehara Tauberian theorem setting there $c_{k}=k^{-\rho+1} b_{k}, k \geq 1$, and

$$
\tilde{D}(s)=\sum_{k \geq 1} b_{k} k^{-s-\rho+1}=D(s+\rho-1), \quad s=\sigma+i y .
$$

Since both $C_{0}, \rho>0$ from condition $\left(M_{1}\right)$, the function $\tilde{D}(s)$ satisfies the condition of Wiener-Ikehara theorem with $c=A$. Moreover, since by (2.9) $\alpha_{n}^{-1} \rightarrow \infty$ as $n \rightarrow \infty$, we can apply (2.14) in the form

$$
\sum_{1 \leq k \leq K^{\prime} / \alpha_{n}} k^{-\rho+1} b_{k} \sim A K / \alpha_{n}, \quad n \rightarrow \infty
$$

where the constant $K>0$ will be specified later. Our next argument will be similar to that given in [8; Lemma 7]. First, using (2.15), we observe that $\|u k\|=u k$ if $|u| k<1 / 2$. This implies that, for $1 \leq k \leq \pi / \alpha_{n},\|u k\|$ can be replaced by $|u| k$. Recalling that $|u| \geq \delta_{n} / 2 \pi$ and applying (2.16) and (2.11), 
we obtain

$$
\begin{aligned}
& S_{n}=\sum_{k=1}^{\infty} b_{k} e^{-k \alpha_{n}} \sin ^{2}(\pi u k) \geq 4 \sum_{k=1}^{\infty} b_{k} e^{-k \alpha_{n}}\|u k\|^{2} \\
& \geq 4 u^{2} \sum_{1 \leq k \leq \pi / \alpha_{n}} k^{2} b_{k} e^{-k \alpha_{n}} \geq\left(\delta_{n} / \pi\right)^{2} \sum_{1 \leq k \leq \pi / \alpha_{n}} k^{2} b_{k} e^{-k \alpha_{n}} \\
& =\frac{\alpha_{n}^{2+2 \rho / 3}}{(\pi \omega(n))^{2}} \sum_{1 \leq k \leq \pi / \alpha_{n}} k^{\rho+1} \frac{k^{2} b_{k}}{k^{\rho+1}} e^{-k \alpha_{n}} \\
& =\frac{\alpha_{n}^{2+2 \rho / 3}}{(\pi \omega(n))^{2}} \sum_{1 \leq k \leq \pi / \alpha_{n}}\left(k^{\rho+1} e^{-k \alpha_{n}}\right)\left(b_{k} k^{-\rho+1}\right) .
\end{aligned}
$$

It is easy to check that the sequence $k^{\rho+1} e^{-k \alpha_{n}}, k \geq 1$, is non-increasing if $k \geq(\rho+1) / \alpha_{n}-1 / 2+O\left(\alpha_{n}\right)$. Hence, for $\rho+1<\pi$, 2.20) with $K=\pi, \rho+1$ implies that

$$
\begin{aligned}
& S_{n} \geq \frac{\alpha_{n}^{2+2 \rho / 3}}{(\pi \omega(n))^{2}} \sum_{(\rho+1) / \alpha_{n} \leq k \leq \pi / \alpha_{n}}\left(k^{\rho+1} e^{-k \alpha_{n}}\right)\left(b_{k} k^{-\rho+1}\right) \\
& \geq \frac{\alpha_{n}^{2+2 \rho / 3}}{(\pi \omega(n))^{2}}\left(\pi / \alpha_{n}\right)^{\rho+1} e^{-\pi} \sum_{(\rho+1) / \alpha_{n} \leq k \leq \pi / \alpha_{n}} b_{k} k^{-\rho+1} \\
& =\frac{\pi^{\rho-1} e^{-\pi} \alpha_{n}^{1-\rho / 3}}{\omega^{2}(n)}\left(\sum_{1 \leq k \leq \pi / \alpha_{n}} b_{k} k^{-\rho+1}-\sum_{1 \leq k<(\rho+1) / \alpha_{n}} b_{k} k^{-\rho+1}\right) \\
& \sim A \pi^{\rho-1}(\pi-\rho-1) e^{-\pi} \alpha_{n}^{-\rho / 3} / \omega^{2}(n) .
\end{aligned}
$$

If $\pi \leq \rho+1$, then $k^{\rho+1} e^{-k \alpha_{n}}$ is a non-decreasing sequence for $1 \leq k \leq \pi / \alpha_{n}$. Then, for some $l \in(0, \pi), k^{\rho+1} e^{-k \alpha_{n}} \geq l^{\rho+1} e^{-l \alpha_{n}}$ and in the same way we observe that

$$
\begin{aligned}
& S_{n} \geq \frac{\alpha_{n}^{2+2 \rho / 3}}{(\pi \omega(n))^{2}} \sum_{l / \alpha_{n} \leq k \leq \pi / \alpha_{n}}\left(k^{\rho+1} e^{-k \alpha_{n}}\right)\left(b_{k} k^{-\rho+1}\right) \\
& \geq \frac{\alpha_{n}^{2+2 \rho / 3} l^{\rho+1} e^{-l}}{(\pi \omega(n))^{2} \alpha_{n}^{\rho+1}} \sum_{l / \alpha_{n} \leq k \leq \pi / \alpha_{n}} b_{k} k^{-\rho+1} \\
& \sim \frac{A l^{\rho+1} e^{-l}(\pi-l) \alpha_{n}^{-\rho / 3}}{(\pi \omega(n))^{2}}(1+o(1)) .
\end{aligned}
$$

Consequently, (2.18), (2.21) and (2.22) imply that there are two constants $C_{3}, \epsilon_{1}>0$, such that

$$
S_{n} \geq C_{3} \alpha_{n}^{-\epsilon_{1}}
$$

uniformly for $\delta_{n} / 2 \pi \leq|u|<1 / 2$. Moreover, $\epsilon_{1} \leq \min (\epsilon, \rho / 3)$ since $\omega(n) \rightarrow \infty$ as $n \rightarrow \infty$ arbitrarily slowly. Hence, noting that $u=\theta / 2 \pi$, we obtain that the required inequality holds uniformly for $\delta_{n} \leq|\theta|<\pi$. 
We now recall (2.6) from Lemma 2. It implies that

$$
\mathcal{B}_{b}^{1 / 2}\left(e^{-\alpha_{n}}\right) \sim(h(\rho+1))^{1 / 2} \alpha_{n}^{-1-\rho / 2}, \quad n \rightarrow \infty .
$$

Combining this asymptotic equivalence with the result of Lemma 4, we obtain Hayman "decay" condition [7; p. 565], namely,

$$
\left|f_{b}\left(e^{-\alpha_{n}+i \theta}\right)\right|=o\left(f_{b}\left(e^{-\alpha_{n}}\right) / \mathcal{B}_{b}^{1 / 2}\left(e^{-\alpha_{n}}\right)\right), \quad n \rightarrow \infty,
$$

uniformly for $\delta_{n} \leq|\theta|<\pi$.

Eqs. (2.10), (2.12) and (2.24) show that the function $f_{b}(x)$ is admissible in the sense of Hayman. Therefore, we can apply Thm. VIII.4 of [7] for its coefficients. We state this result in the next lemma.

Lemma 5 Suppose that the weight sequence $b$ satisfies Meinardus conditions $\left(M_{1}\right)-\left(M_{3}\right)$. Then, the asymptotic for the total number of weighted partitions is given by

$$
p_{b}(n) \sim \frac{e^{n \alpha_{n}} f_{b}\left(e^{-\alpha_{n}}\right)}{\sqrt{2 \pi \mathcal{B}_{b}\left(e^{-\alpha_{n}}\right)}}
$$

as $n \rightarrow \infty$, where $\alpha_{n}$ is the unique solution of (2.8) whose asymptotic expansion is given by (2.9) and $\mathcal{B}_{b}\left(e^{-\alpha_{n}}\right)$ is defined by (2.6).

Remark. The asymptotic equivalence (2.25) is in fact Meinardus asymptotic formula [13] for the number of weighted partitions of $n$. Here we give the formula in a slightly different form, which is more convenient for our further asymptotic analysis. One can easily show the coincidence of (2.25) with the Meinardus original formula, applying the result of Lemma 1 to $f_{b}\left(e^{-\alpha_{n}}\right)$ and replacing $\alpha_{n}$ and $\mathcal{B}_{b}\left(e^{-\alpha_{n}}\right)$ by (2.9) and (2.6), respectively.

Further, we also need a bound on the rate of growth of the weights $b_{k}$, as $k \rightarrow \infty$. Using Wiener-Ikehara Tauberian theorem, Granovsky et al. [10; p. 310] showed that $b_{k}=o\left(k^{\rho}\right)$ as $k \rightarrow \infty$. We need this bound in a different slightly more precise form.

Lemma 6 If the sequence of weights $b$ satisfies conditions $\left(M_{1}\right)$ and $\left(M_{2}\right)$, then there is a sequence of numbers $L_{k}, k \geq 1$, satisfying $\lim _{k \rightarrow \infty} L_{k}=0$ and such that

$$
b_{k}=\left(L_{k}-L_{k-1}\right) k^{\rho}+\left(A+L_{k-1}\right) k^{\rho-1}, \quad k \geq 2, \quad L_{1}=b_{1}-A,
$$

where $A$ is the constant defined in condition $\left(M_{1}\right)$.

Proof. As in [10; p. 310], we rewrite (2.14) in the following way:

$$
\frac{1}{k} \sum_{j=1}^{k} c_{j}=\frac{1}{k} c_{k}+\frac{1}{k} \sum_{j=1}^{k-1} c_{j}=c+L_{k}, \quad k \geq 2,
$$


where $\lim _{k \rightarrow \infty} L_{k}=0$. We also set $L_{1}=c_{1}-c$. Then, for $k \geq 2$, we have

$$
\frac{1}{k} c_{k}=L_{k}-L_{k-1}+\frac{1}{k}\left(c+L_{k-1}\right) \text {. }
$$

To obtain (2.26) it remains to set $c_{k}=k^{-\rho+1} b_{k}, k \geq 1, c=A$ and recall that the weights $b_{k}$ satisfy conditions $\left(M_{1}\right)$ and $\left(M_{2}\right)$ with $C_{0}, \rho>0$.

Now, we also recall formula (1.2) for the truncated products $f_{m, b}(x), m \geq 1$. Similarly to (2.1), we set

$$
F_{m, b}(x)=\log f_{m, b}(x)=-\sum_{k=1}^{m} b_{k} \log \left(1-x^{k}\right) .
$$

(Here we consider the main branch of the logarithmic function, assuming that $\log y<0$ for $0<y<1$ ). Further on, when computing the derivatives of (2.1) and (2.27), we shall write

$$
F_{b}^{(j)}\left(e^{-\alpha_{n}}\right)=\left.F_{b}^{(j)}(x)\right|_{x=e^{-\alpha_{n}},} F_{m, b}^{(j)}\left(e^{-\alpha_{n}}\right)=\left.F_{m, b}^{(j)}(x)\right|_{x=e^{-\alpha_{n}}, j=1,2,3 .} .
$$

Our next lemma establishes estimates on the tails $F_{b}^{(j)}\left(e^{-\alpha_{n}}\right)-F_{m, b}^{(j)}\left(e^{-\alpha_{n}}\right)$ for some specific values of $m$.

Lemma 7 Suppose that the weight sequence $b$ satisfies conditions $\left(M_{1}\right)$ and $\left(M_{2}\right)$ and that $\alpha_{n}, n \geq 1$, is defined by eq. (2.9). Moreover, let $m=m(n)$ be a sequence of integers satisfying

$$
m \sim \rho \alpha_{n}^{-1} \log \alpha_{n}^{-1}, \quad n \rightarrow \infty .
$$

Then

$$
F_{b}^{(j)}\left(e^{-\alpha_{n}}\right)-F_{m, b}^{(j)}\left(e^{-\alpha_{n}}\right)=O\left(\alpha_{n}^{-j} \log ^{\rho+j} \alpha_{n}^{-1}\right), \quad j=1,2,3 .
$$

Proof. We shall consider only the case $j=1$. The other two cases are studied in a similar way.

First, we choose a sequence of integers $m_{1}(n)$ that satisfies the asymptotic equivalence

$$
m_{1}=m_{1}(n) \sim(\rho+1) \alpha_{n}^{-1} \log \alpha_{n}^{-1}
$$

and decompose the difference of the first derivatives in the following way:

$$
F_{b}^{\prime}\left(e^{-\alpha_{n}}\right)-F_{m, b}^{\prime}\left(e^{-\alpha_{n}}\right)=\sum_{k=m+1}^{\infty} \frac{k b_{k} e^{-(k-1) \alpha_{n}}}{1-e^{-k \alpha_{n}}}=\Sigma_{1}+\Sigma_{2},
$$

where

$$
\Sigma_{1}=\sum_{k=m+1}^{m_{1}} \frac{k b_{k} e^{-(k-1) \alpha_{n}}}{1-e^{-k \alpha_{n}}}, \quad \Sigma_{2}=\sum_{k=m_{1}+1}^{\infty} \frac{k b_{k} e^{-(k-1) \alpha_{n}}}{1-e^{-k \alpha_{n}}} .
$$

We also notice that (2.28) and (2.29) imply that

$$
e^{-m \alpha_{n}} \sim \alpha_{n}^{\rho}, \quad e^{-m_{1} \alpha_{n}} \sim \alpha_{n}^{\rho+1}, \quad n \rightarrow \infty .
$$


Hence, applying the result of Lemma 6 , for $\Sigma_{1}$ we obtain

$$
\begin{aligned}
& \Sigma_{1}=O\left(\alpha_{n}^{\rho} \sum_{k=m+1}^{m_{1}} k b_{k}\right) \\
& =O\left(\alpha_{n}^{\rho} \sum_{k=m+1}^{m_{1}} k^{\rho+1}\left(L_{k}-L_{k-1}\right)\right)+O\left(\alpha_{n}^{\rho} \sum_{k=m+1}^{m_{1}} k^{\rho}\right) .
\end{aligned}
$$

The second summand in (2.32) can be approximated by a Riemann integral using (2.28) and (2.29). We have

$$
\sum_{k=m+1}^{m_{1}} k^{\rho}=m_{1}^{\rho+1} \sum_{\frac{\rho}{\rho+1}<\frac{k}{m_{1}} \leq 1}\left(\frac{k}{m_{1}}\right)^{\rho} \frac{1}{m_{1}} \sim m_{1}^{\rho+1} \int_{\frac{\rho}{\rho+1}}^{1} u^{\rho} d u=O\left(m_{1}^{\rho+1}\right) .
$$

Therefore

$$
O\left(\alpha_{n}^{\rho} \sum_{k=m+1}^{m_{1}} k^{\rho}\right)=O\left(\alpha_{n}^{\rho} m_{1}^{\rho+1}\right)=O\left(\alpha_{n}^{-1} \log ^{\rho+1} \alpha_{n}^{-1}\right) .
$$

The first sum in (2.32) can be also rewritten as

$$
\begin{aligned}
& \sum_{k=m+1}^{m_{1}} k^{\rho+1}\left(L_{k}-L_{k-1}\right)=-L_{m}(m+1)^{\rho+1}+L_{m_{1}} m_{1}^{\rho+1} \\
& +\sum_{j=1}^{m_{1}-m-1} L_{m+j}\left((m+j)^{\rho+1}-(m+j+1)^{\rho+1}\right) .
\end{aligned}
$$

We recall that by Lemma 6, (2.28) and (2.29), $L_{m}=o(1)$ and $L_{m_{1}}=o(1)$. Hence

$$
\left(-L_{m}(m+1)^{\rho+1}+L_{m_{1}} m_{1}^{\rho+1}\right) \alpha_{n}^{\rho}=o\left(\alpha_{n}^{-1} \log ^{\rho+1} \alpha_{n}^{-1}\right) .
$$

The last sum in (2.34) is estimated using again an approximation by an integral. First, applying a binomial expansion, we get

$$
\begin{aligned}
& (m+j)^{\rho+1}-(m+j+1)^{\rho+1}=(m+j)^{\rho+1}\left(1-\left(1+\frac{1}{m+j}\right)^{\rho+1}\right) \\
& =(m+j)^{\rho+1}\left(-\frac{\rho+1}{m+j}+O\left((m+j)^{-2}\right)\right) \\
& =-(\rho+1)(m+j)^{\rho}+O\left((m+j)^{\rho-1}\right) .
\end{aligned}
$$


Then, from (2.28), (2.29) and the fact that $L_{m+j}$ are bounded for $1 \leq j \leq$ $m_{1}-m-1$ we obtain

$$
\begin{aligned}
& \sum_{j=1}^{m_{1}-m-1} L_{m+j}\left((m+j)^{\rho+1}-(m+j+1)^{\rho+1}\right) \\
= & -(\rho+1) \sum_{j=1}^{m_{1}-m-1} L_{m+j}\left((m+j)^{\rho}+O\left((m+j)^{\rho-1}\right)\right) \\
= & -(\rho+1) m_{1}^{\rho+1} \sum_{\frac{j}{m_{1}}<1-\frac{m}{m_{1}}} L_{m+j}\left(\frac{1}{m_{1}}\right)\left(\left(\frac{m+j}{m_{1}}\right)^{\rho}+O\left(\frac{1}{m_{1}}\left(\frac{m+j}{m_{1}}\right)^{\rho-1}\right)\right) \\
= & O\left(m_{1}^{\rho+1} \int_{0}^{1-\frac{\rho}{\rho+1}}\left(\left(\frac{\rho}{\rho+1}+u\right)^{\rho}+O\left(\frac{1}{m_{1}}\right)\right) d u\right)=O\left(m_{1}^{\rho+1}\right) .
\end{aligned}
$$

Combining (2.29) with (2.32)-(2.36), we obtain

$$
\Sigma_{1}=O\left(\alpha_{n}^{-1} \log ^{\rho+1} \alpha_{n}^{-1}\right) .
$$

$\Sigma_{2}$ can be estimated in a similar way. We have

$$
\begin{aligned}
& \Sigma_{2}=O\left(\sum_{k=m_{1}+1}^{\infty} \frac{k^{\rho+1} e^{-k \alpha_{n}}}{1-e^{-k \alpha_{n}}}\right)=O\left(\sum_{k=m_{1}+1}^{\infty} k^{\rho+1} e^{-k \alpha_{n}}\right) \\
& =O\left(\alpha_{n}^{-\rho-2} \int_{m_{1} \alpha_{n}}^{\infty} u^{\rho+1} e^{-u} d u\right)=O\left(\alpha_{n}^{-\rho-2}\left(m_{1} \alpha_{n}\right)^{\rho+1} e^{-m_{1} \alpha_{n}}\right) \\
& =O\left(\alpha_{n}^{-1} \log ^{\rho+1} \alpha_{n}^{-1}\right)
\end{aligned}
$$

where the last equality follows from (2.29) and (2.31), while in the previous one we have used the the asymptotic behavior of the incomplete gamma function $\Gamma(a, z)$ as $z \rightarrow \infty$ (see [1; Sect. 6.5]). The required estimate now follows from (2.30), (2.37) and (2.38).

Our last forthcoming lemma supplies us with integral representations for $F_{b}\left(e^{-\alpha}\right)$ and $F_{m, b}\left(e^{-\alpha}\right), \alpha>0$, using the Dirichlet series (1.3) and its partial sums

$$
D_{m}(s)=\sum_{k=1}^{m} b_{k} k^{-s}, \quad s=\sigma+i y, \quad m \geq 1 .
$$

The proof is based on a Mellin transform technique and can be found in [13], [10; Lemma 2(ii)] and [2; Sect. 6.2].

Lemma 8 For any $\alpha, \Delta>0$, we have

$$
F_{m, b}\left(e^{-\alpha}\right)=\frac{1}{2 \pi i} \int_{\rho+\Delta-i \infty}^{\rho+\Delta+i \infty} \alpha^{-s} \Gamma(s) \zeta(s+1) D_{m}(s) d s
$$

and

$$
F_{b}\left(e^{-\alpha}\right)=\frac{1}{2 \pi i} \int_{\rho+\Delta-i \infty}^{\rho+\Delta+i \infty} \alpha^{-s} \Gamma(s) \zeta(s+1) D(s) d s,
$$

where $D_{m}(s)$ and $D(s)$ are defined by (2.39) and (1.3), respectively. 


\section{Proof of the Main Result}

We apply first the Cauchy coefficient formula to (1.2) using the circle $x=$ $e^{-\alpha_{n}+i \theta}, \pi<\theta \leq \pi$, as a contour of integration $\left(\alpha_{n}\right.$ is determined by (2.9) $)$. We obtain

$$
p_{b}(n) \mathbb{P}\left(X_{n} \leq m\right)=\frac{e^{n \alpha_{n}}}{2 \pi} \int_{-\pi}^{\pi} f_{m, b}\left(e^{-\alpha_{n}+i \theta}\right) e^{-i \theta n} d \theta .
$$

Then, we break up the range of integration as follows:

$$
p_{b}(n) \mathbb{P}\left(X_{n} \leq m\right)=J_{1}(m, n)+J_{2}(m, n),
$$

where

$$
\begin{gathered}
J_{1}(m, n)=\frac{e^{n \alpha_{n}}}{2 \pi} \int_{-\delta_{n}}^{\delta_{n}} f_{m, b}\left(e^{-\alpha_{n}+i \theta}\right) e^{-i \theta n} d \theta \\
=\frac{e^{n \alpha_{n}+F_{m, b}\left(e^{-\alpha_{n}}\right)}}{2 \pi} \int_{-\delta_{n}}^{\delta_{n}} \frac{f_{m, b}\left(e^{-\alpha_{n}+i \theta}\right)}{f_{m, b}\left(e^{-\alpha_{n}}\right)} e^{-i \theta n} d \theta, \\
J_{2}(m, n)=\frac{e^{n \alpha_{n}+F_{m, b}\left(e^{-\alpha_{n}}\right)}}{2 \pi} \int_{\delta_{n}<|\theta| \leq \pi} \frac{f_{m, b}\left(e^{-\alpha_{n}+i \theta}\right)}{f_{m, b}\left(e^{-\alpha_{n}}\right)} e^{-i \theta n} d \theta
\end{gathered}
$$

( $\delta_{n}$ and $F_{m, b}(x)$ are defined by (2.11) and (2.27), respectively).

We start with an estimate for $J_{1}(m, n)$, expanding the integrand of (3.2) by Taylor formula:

$$
\begin{aligned}
& \frac{f_{m, b}\left(e^{-\alpha_{n}+i \theta}\right)}{f_{m, b}\left(e^{-\alpha_{n}}\right)}=\exp \left\{\left(e^{i \theta}-1\right) e^{-\alpha_{n}} F_{m, b}^{\prime}\left(e^{-\alpha_{n}}\right)\right. \\
& \left.+\frac{1}{2}\left(e^{i \theta}-1\right)^{2} e^{-2 \alpha_{n}} F_{m, b}^{\prime \prime}\left(e^{-\alpha_{n}}\right)+O\left(|\theta|^{3} F_{m, b}^{\prime \prime \prime}\left(e^{-\alpha_{n}}\right)\right)\right\} .
\end{aligned}
$$

Hence, we can rewrite (3.2) as follows:

$$
J_{1}(m, n)=\frac{e^{n \alpha_{n}}}{\sqrt{2 \pi}} e^{F_{m, b}\left(e^{-\alpha_{n}}\right)} I_{n}
$$

where

$$
\begin{aligned}
& I_{n}=\frac{1}{\sqrt{2 \pi}} \int_{-\delta_{n}}^{\delta_{n}} \exp \left\{\left(e^{i \theta}-1\right) e^{-\alpha_{n}} F_{m, b}^{\prime}\left(e^{-\alpha_{n}}\right)\right. \\
& \left.+\frac{1}{2}\left(e^{i \theta}-1\right)^{2} e^{-2 \alpha_{n}} F_{m, b}^{\prime \prime}\left(e^{-\alpha_{n}}\right)+O\left(|\theta|^{3} F_{m, b}^{\prime \prime \prime}\left(e^{-\alpha_{n}}\right)\right)-i \theta n\right\} d \theta .
\end{aligned}
$$

Lemma 7 shows that, for those integers $m$ satisfying (2.28), we can replace the derivatives $F_{m, b}^{(j)}\left(e^{-\alpha_{n}}\right)$ by $F_{b}^{(j)}\left(e^{-\alpha_{n}}\right), j=1,2,3$, at the expense of a negligible error term. In fact, combining Lemma 7 with (2.11), we have

$$
\begin{aligned}
& \left(e^{i \theta}-1\right)^{j} F_{m, b}^{(j)}\left(e^{-\alpha_{n}}\right)=\left(e^{i \theta}-1\right)^{j} F_{b}^{(j)}\left(e^{-\alpha_{n}}\right)+O\left(\delta_{n}^{j} \alpha_{n}^{-j} \log ^{\rho+j} \alpha_{n}^{-1}\right) \\
& =\left(e^{i \theta}-1\right)^{j} F_{b}^{(j)}\left(e^{-\alpha_{n}}\right)+O\left(\alpha_{n}^{\rho j / 3} \log ^{\rho+j} \alpha_{n}^{-1} / \omega^{j}(n)\right), \quad j=1,2,
\end{aligned}
$$


and

$$
O\left(|\theta|^{3} F_{m, b}^{\prime \prime \prime}\left(e^{-\alpha_{n}}\right)\right)=O\left(|\theta|^{3} F_{b}^{\prime \prime \prime}\left(e^{-\alpha_{n}}\right)\right)+O\left(\alpha_{n}^{\rho}\left(\log ^{\rho+3} \alpha_{n}^{-1}\right) / \omega^{3}(n)\right),
$$

where the function $\omega(n) \rightarrow \infty$ as $n \rightarrow \infty$ arbitrarily slowly. Since all error terms above tend to 0 , we obtain

$$
\begin{aligned}
& I_{n}=\frac{1+o(1)}{\sqrt{2 \pi}} \int_{-\delta_{n}}^{\delta_{n}} \exp \left\{F_{b}\left(e^{-\alpha_{n}+i \theta}\right)-F_{b}\left(e^{-\alpha_{n}}\right)-i \theta n\right\} d \theta \\
& =\frac{1+o(1)}{\sqrt{2 \pi}} \int_{-\delta_{n}}^{\delta_{n}} \frac{f_{b}\left(e^{-\alpha_{n}+i \theta}\right)}{f_{b}\left(e^{-\alpha_{n}}\right)} e^{-i \theta n} d \theta
\end{aligned}
$$

where the last equality follows from a similar Taylor expansion for $F_{b}\left(e^{-\alpha_{n}+i \theta}\right)$ and the fact that $O\left(|\theta|^{3} F_{b}^{\prime \prime \prime}\left(e^{-\alpha_{n}}\right)\right)=O\left(\delta_{n}^{3} F_{b}^{\prime \prime \prime}\left(e^{-\alpha_{n}}\right)\right)=O\left(1 / \omega^{3}(n)\right)=o(1)$ (see (2.7) of Lemma 2 and (2.11)). Now, from Lemma 3 it follows that

$$
\begin{aligned}
& I_{n} \sim \frac{1}{\sqrt{2 \pi}} \int_{-\delta_{n}}^{\delta_{n}} e^{-\theta^{2} \mathcal{B}_{b}\left(e^{-\alpha_{n}}\right) / 2} d \theta \\
& =\frac{1}{\sqrt{2 \pi \mathcal{B}_{b}\left(e^{-\alpha_{n}}\right)}} \int_{-\delta_{n} \sqrt{\mathcal{B}_{b}\left(e^{-\alpha_{n}}\right)}}^{\delta_{n} \sqrt{\mathcal{B}_{b}\left(e^{-\alpha_{n}}\right)}} e^{-y^{2} / 2} d y \sim \frac{1}{\sqrt{2 \pi \mathcal{B}_{b}\left(e^{-\alpha_{n}}\right)}} \int_{-\infty}^{\infty} e^{-y^{2} / 2} d y \\
& =\frac{1}{\sqrt{\mathcal{B}_{b}\left(e^{-\alpha_{n}}\right)}}, \quad n \rightarrow \infty .
\end{aligned}
$$

The last asymptotic equivalence follows from (2.6) of Lemma 2, which implies that

$$
\delta_{n} \sqrt{\mathcal{B}_{b}\left(e^{-\alpha_{n}}\right)} \sim \frac{\alpha_{n}^{-\rho / 6}}{\omega(n)} \sqrt{h(\rho+1)} \rightarrow \infty,
$$

if $\omega(n) \rightarrow \infty$ slower than $\alpha_{n}^{-\rho / 6}$.

Substituting the asymptotic equivalence for $I_{n}$ into (3.4), we conclude that

$$
J_{1}(m, n) \sim \frac{e^{n \alpha_{n}}}{\sqrt{2 \pi \mathcal{B}\left(e^{-\alpha_{n}}\right)}} e^{F_{m, b}\left(e^{-\alpha_{n}}\right)}
$$

if $m$ satisfies (2.28) as $n \rightarrow \infty$.

For the estimate of $J_{2}(m, n)$, we recall (3.3) and the proof of (2.18). Thus, for any real $u$, we obtain

$$
\begin{aligned}
& \Re\left(F_{m, b}\left(e^{-\alpha_{n}+2 \pi i u}\right)\right)-F_{m, b}\left(e^{-\alpha_{n}}\right) \leq-\frac{\log 5}{2} \sum_{k=1}^{m} b_{k} e^{-\alpha_{n} k} \sin ^{2}(\pi u k) \\
& =-\frac{\log 5}{2}\left(\sum_{k=1}^{\infty} b_{k} e^{-\alpha_{n} k} \sin ^{2}(\pi u k)-\sum_{k=m+1}^{\infty} b_{k} e^{-\alpha_{n} k} \sin ^{2}(\pi u k)\right) .
\end{aligned}
$$


Consider again the sequences $m=m(n)$ and $m_{1}=m_{1}(n)$ defined by (2.28) and (2.29), respectively. We have

$$
\begin{aligned}
& \sum_{k=m+1}^{\infty} b_{k} e^{-\alpha_{n} k} \sin ^{2}(\pi k u) \leq \sum_{k=m+1}^{\infty} b_{k} e^{-\alpha_{n} k} \\
= & \sum_{k=m+1}^{m_{1}} b_{k} e^{-\alpha_{n} k}+\sum_{k=m_{1}+1}^{\infty} b_{k} e^{-\alpha_{n} k}
\end{aligned}
$$

These sums can be estimated using the argument given in the proof of Lemma 7 (see (2.32)-(2.38) ). We obtain in the same way that

$$
\begin{gathered}
\sum_{k=m+1}^{m_{1}} b_{k} e^{-\alpha_{n} k}=O\left(\alpha_{n}^{\rho} \sum_{k=m+1}^{m_{1}} b_{k}\right)=O\left(\left(\alpha_{n} m_{1}\right)^{\rho}\right)=O\left(\log ^{\rho} \alpha_{n}^{-1}\right), \\
\sum_{k=m_{1}+1}^{\infty} b_{k} e^{-\alpha_{n} k}=O\left(\sum_{k=m_{1}+1}^{\infty} k^{\rho} e^{-\alpha_{n} k}\right)=O\left(\log ^{\rho} \alpha_{n}^{-1}\right)
\end{gathered}
$$

and thus, (3.7)-(3.9) imply that

$$
\sum_{k=m+1}^{\infty} b_{k} e^{-\alpha_{n} k} \sin ^{2}(\pi k u)=O\left(\log ^{\rho} \alpha_{n}^{-1}\right) .
$$

Replacing the second term of the right-hand side of (3.6) by the last $O$-estimate and applying inequality (2.23) (see also (1.6)) to its first term, for $\delta_{n} / 2 \pi \leq 1$ $u \mid<1 / 2$, we obtain

$$
\Re\left(F_{m, b}\left(e^{-\alpha_{n}+2 \pi i u}\right)\right)-F_{m, b}\left(e^{-\alpha_{n}}\right) \leq-C_{3} \alpha_{n}^{-\epsilon_{1}}+O\left(\log ^{\rho} \alpha_{n}^{-1}\right) .
$$

Now, we are ready to compare the growth of (3.3) with that of (3.5) whenever $m$ satisfies (2.28). We have

$$
\begin{aligned}
& \left|J_{2}(m, n)\right| \leq \exp \left(n \alpha_{n}+F_{m, b}\left(e^{-\alpha_{n}}\right)\right) \\
& \times \int_{\frac{\delta_{n}}{2 \pi}<|u| \leq \frac{1}{2}}\left|f_{m, b}\left(e^{-\alpha_{n}+2 \pi i u}\right) / f_{m, b}\left(e^{-\alpha_{n}}\right)\right| d u \\
& =\exp \left(n \alpha_{n}+F_{m, b}\left(e^{-\alpha_{n}}\right)\right) \int_{\frac{\delta_{n}}{2 \pi}<|u| \leq \frac{1}{2}}\left(\Re\left(F_{m, b}\left(e^{-\alpha_{n}+2 \pi i u}\right)-F_{m, b}\left(e^{-\alpha_{n}}\right)\right) d u\right. \\
& =O\left(\exp \left(n \alpha_{n}+F_{m, b}\left(e^{-\alpha_{n}}\right)-C_{3} \alpha_{n}^{-\epsilon_{1}}+O\left(\log ^{\rho} \alpha_{n}^{-1}\right)\right)\right) \\
& =O\left(e^{-C_{3} \alpha_{n}^{-\epsilon_{1}}} \sqrt{2 \pi \mathcal{B}_{b}\left(e^{-\alpha_{n}}\right)} J_{1}(m, n)\right)=o\left(J_{1}(m, n)\right)
\end{aligned}
$$

where for the last $o$-estmate we have used (2.6). It is now clear that (3.1), (3.5) and (3.10) imply that

$$
p_{b}(n) \mathbb{P}\left(X_{n} \leq m\right) \sim \frac{e^{n \alpha_{n}}}{\sqrt{2 \pi \mathcal{B}_{b}\left(e^{-\alpha_{n}}\right)}} e^{F_{m, b}\left(e^{-\alpha_{n}}\right)}, \quad n \rightarrow \infty .
$$


Subsequent application of the asymptotic equivalence (2.25) from Lemma 5 implies that

$$
\mathbb{P}\left(X_{n} \leq m\right) \sim \exp \left\{F_{m, b}\left(e^{-\alpha_{n}}\right)-F_{b}\left(e^{-\alpha_{n}}\right)\right\},
$$

where $\alpha_{n}$ and $m$ satisfy (2.9) and (2.28), respectively.

Further on we shall study the asymptotic behavior of the exponent in (3.11). Our analysis will be based on a generalization of Perron formula that expresses the partial sums of a Dirichlet series as complex integrals of the inverse Mellin type transforms applied to the Dirichlet series itself. We shall use it in the form given in the Supplement of [18; Sect. 3]. So, first we represent $F_{m, b}\left(e^{-\alpha_{n}}\right)$ using eq. (2.40) of Lemma 8 and then, we apply Perron formula to the partial sum $D_{m}(s)$ of the Dirichlet series $D(s)$ (recall also (2.39) and (1.3)). In this way we arrive at the following complex integral representation: for any $\Delta>1$, we have

$$
\begin{aligned}
& F_{m, b}\left(e^{-\alpha_{n}}\right) \\
& =\frac{1}{2 \pi i} \int_{\rho+\Delta-i \infty}^{\rho+\Delta+i \infty} \alpha_{n}^{-s} \Gamma(s) \zeta(s+1)\left(\frac{A(m+1)^{\rho-s}}{\rho-s}+D(s)+\Omega_{m}\right) d s
\end{aligned}
$$

where $\Omega_{m}=o(1), m \rightarrow \infty$. Furthermore, (3.11) and (3.12) imply that

$$
\begin{aligned}
& \mathbb{P}\left(X_{n} \leq m\right) \\
& \sim \exp \left\{-\frac{A \alpha_{n}^{-\rho}}{2 \pi i} \int_{\Delta-i \infty}^{\Delta+i \infty}\left((m+1) \alpha_{n}\right)^{-s} \Gamma(s+\rho) \zeta(s+\rho+1) \frac{d s}{s}\right\} .
\end{aligned}
$$

The proofs of (3.12) and (3.13) contain some technical details that will be given in the Appendix.

We continue with the computation of the complex integral in the exponent of (3.13). The sequence $m=m(n)$ will be specified later in a more precise way. At this moment we only asume that it satisfies (2.28). We set in the integral of (3.13)

$$
u=u_{n}=(m+1) \alpha_{n},
$$

and consider it as a function of $u$. First, we shall obtain its explicit form and then we shall estimate it as $u \rightarrow \infty$ (see (2.28) and (3.14) $)$. Clearly, we can consider this integral as the inverse Mellin transform of the function $\Gamma(s+\rho) \zeta(s+\rho+1) / s$. For the sake of convenience, we set

$$
H(u)=\frac{1}{2 \pi i} \int_{\Delta-i \infty}^{\Delta+i \infty} u^{-s} \Gamma(s+\rho) \zeta(s+\rho+1) \frac{d s}{s} .
$$

It is known that, for $\Re(s)>0, g_{1}(s)=1 / s$ is the Mellin transform of the (Heaviside-like) step function

$$
H_{1}(u)=\left\{\begin{array}{lll}
1 & \text { if } & 0 \leq u<1 \\
0 & \text { if } & u>1
\end{array}\right.
$$

while $g_{2}(s)=\Gamma(s) \zeta(s+1)$ is the Mellin transform of

$$
H_{2}(u)=\sum_{j=1}^{\infty} \frac{e^{-j u}}{j}=-\log \left(1-e^{-u}\right)
$$


(see e.g. [7; Appendix B.7]). Next, for $\Delta>1$, we apply formula (6.1.14) from [5] with $\alpha=0$ and $\beta=\rho-1$. We obtain

$$
\begin{aligned}
& H(u)=u^{\alpha} \int_{0}^{\infty} y^{\beta} H_{1}(u / y) H_{2}(y) d y=-\int_{u}^{\infty} y^{\rho-1} \log \left(1-e^{-y}\right) d y \\
& =\int_{u}^{\infty} y^{\rho-1} e^{-y} d y+R(u)=\Gamma(\rho, u)+R(u),
\end{aligned}
$$

where $\Gamma(\rho, u)$ denotes the incomplete gamma function, while $R(u)$ is the error term given by

$$
R(u)=\int_{u}^{\infty} y^{\rho-1}\left(\sum_{j=2}^{\infty} \frac{e^{-j y}}{j}\right) d y .
$$

It is easily estimated as follows:

$$
R(u) \leq \frac{1}{2\left(1-e^{-u}\right)} \int_{u}^{\infty} y^{\rho-1} e^{-2 y} d y=O\left(e^{-u} \Gamma(\rho, u)\right), \quad u \rightarrow \infty .
$$

Combining this estimate with (3.13)-(3.15) and (3.17) and applying the asymptotic $\Gamma\left(\rho, u_{n}\right)=u_{n}^{\rho-1} e^{-u_{n}}\left(1+O\left(1 / u_{n}\right)\right)$ of the incomplete gamma function (see again [1; Sect. 6.5]), we obtain

$$
\begin{aligned}
& \mathbb{P}\left(X_{n} \leq m\right) \sim \exp \left\{-A \alpha_{n}^{-\rho}\left(u_{n}^{\rho-1} e^{-u_{n}}\left(1+O\left(1 / u_{n}\right)\right)+O\left(u_{n}^{\rho-1} e^{-2 u_{n}}\right)\right)\right\} \\
& =\exp \left\{-A \alpha_{n}^{-1} m^{\rho-1} e^{-m \alpha_{n}}\left(1+O\left(1 / m \alpha_{n}\right)\right)\right\} \\
& =\exp \left\{-A \alpha_{n}^{-1} m^{\rho-1} e^{-m \alpha_{n}}\left(1+O\left(1 / \log \alpha_{n}^{-1}\right)\right)\right\},
\end{aligned}
$$

where for the last equality we have used again (2.28). It is now clear that $\mathbb{P}\left(X_{n} \leq m\right)$ converges to the distribution function $e^{-e^{-t}},-\infty<t<\infty$, if $m=m(n)$ satisfies

$$
-m \alpha_{n}+(\rho-1) \log m+\log \left(A \alpha_{n}^{-1}\right)=-t+o(1)
$$

as $n \rightarrow \infty$. From this we deduce

$$
m=\alpha_{n}^{-1} \log \alpha_{n}^{-1}+(\rho-1) \alpha_{n}^{-1} \log m+(\log A+t) \alpha_{n}^{-1}+o\left(\alpha_{n}^{-1}\right),
$$

which in turn implies that

$$
\begin{aligned}
& \log m=\log \left(\alpha_{n}^{-1} \log \alpha_{n}^{-1}\right) \\
& +\log \left(1+\frac{\log A+t}{\log \alpha_{n}^{-1}}+(\rho-1) \frac{\log m}{\log \alpha_{n}^{-1}}+o\left(1 / \log \alpha_{n}^{-1}\right)\right) \\
& =\log \alpha_{n}^{-1}+\log \log \alpha_{n}^{-1} \\
& +\log \left(1+\frac{\log A+t}{\log \alpha_{n}^{-1}}+(\rho-1) \frac{\log \alpha_{n}^{-1}+\log \log \alpha_{n}^{-1}+O(1)}{\log \alpha_{n}^{-1}}\right) \\
& =\log \alpha_{n}^{-1}+\log \log \alpha_{n}^{-1}+\log \left(1+(\rho-1)+O\left(\frac{\log \log \alpha_{n}^{-1}}{\log \alpha_{n}^{-1}}\right)\right) \\
& =\log \alpha_{n}^{-1}+\log \log \alpha_{n}^{-1}+\log \rho+O\left(\frac{\log \log \alpha_{n}^{-1}}{\log \alpha_{n}^{-1}}\right) .
\end{aligned}
$$


Hence, (3.19) becomes

$$
\begin{aligned}
& m=\rho \alpha_{n}^{-1} \log \alpha_{n}^{-1}+(\rho-1) \alpha_{n}^{-1} \log \log \alpha_{n}^{-1} \\
& +\alpha_{n}^{-1}(\rho-1) \log \rho+(\log A+t) \alpha_{n}^{-1}+O\left(\alpha_{n}^{-1} \frac{\log \log \alpha_{n}^{-1}}{\log \alpha_{n}^{-1}}\right) .
\end{aligned}
$$

Replacing now this value of $m$ into (3.18) and using the continuity of the distribution function $e^{-e^{-t}},-\infty<t<\infty$, we obtain

$$
\begin{aligned}
& \mathbb{P}\left(X_{n} \leq m\right) \\
& =\mathbb{P}\left(\alpha_{n} X_{n}-\rho \log \alpha_{n}^{-1}-(\rho-1) \log \log \alpha_{n}^{-1}-(\rho-1) \log \rho-\log A+o(1) \leq t\right) \\
& \rightarrow e^{-e^{-t}}, \quad n \rightarrow \infty .
\end{aligned}
$$

To complete the proof of the theorem, it remains to justify the normalization for $X_{n}$ stated in (1.10). We have to show that the sequence $\alpha_{n}, n \geq 1$, in (3.20) can be replaced by $a(n)=a(n ; \rho, A), n \geq 1$ (see (1.9)). So, we recall first (2.9) and notice that taking logarithms from its both sides, we easily obtain

$$
\begin{aligned}
& \log \alpha_{n}^{-1}=|\log a(n)|+O\left(n^{-\frac{\rho}{\rho+1}}\right), \\
& \log \log \alpha_{n}^{-1}=\log |\log a(n)|+O\left(n^{-\frac{\rho}{\rho+1}} / \log n\right) .
\end{aligned}
$$

Next, we set $Z_{n}:=\alpha_{n} X_{n}+z_{n}, Y_{n}:=a(n) X_{n}+y_{n}$, where $z_{n}:=\rho \log \alpha_{n}-(\rho-$ 1) $\log \log \alpha_{n}^{-1}-(\rho-1) \log \rho-\log A, y_{n}=\rho \log a(n)-(\rho-1) \log |\log a(n)|-(\rho-$ 1) $\log \rho-\log A, n \geq 1$. Furthermore, (3.20) can be written in a shorter way as follows:

$$
G_{n, Z}(t):=\mathbb{P}\left(Z_{n} \leq t\right) \rightarrow e^{-e^{-t}}, \quad n \rightarrow \infty .
$$

So, we have to prove the same convergence for $G_{n, Y}(t):=\mathbb{P}\left(Y_{n} \leq t\right), n \geq 1$. It is easy to verify that the above representations for $Y_{n}$ and $Z_{n}$ imply that

$$
Y_{n}=\left(1+\eta_{n}\right) Z_{n}+e_{n},
$$

where $\eta_{n}:=a(n) / \alpha_{n}-1, e_{n}:=y_{n}-\left(a(n) / \alpha_{n}\right) z_{n}, n \geq 1$. From (1.9), (2.9) and (3.21) we obtain the estimates $\eta_{n}=O\left(n^{-\frac{\rho}{\rho+1}}\right)$ and $e_{n}=O\left(n^{-\frac{\rho}{\rho+1}} \log n\right)$ as $n \rightarrow \infty$. Moreover, (3.23) implies that

$$
\begin{aligned}
& G_{n, Y}(t)=\mathbb{P}\left(Z_{n} \leq \frac{t-e_{n}}{1+\eta_{n}}\right)=\mathbb{P}\left(Z_{n} \leq t-\frac{t \eta_{n}+e_{n}}{1+\eta_{n}}\right) \\
& =G_{n, Z}\left(t-\frac{t \eta_{n}+e_{n}}{1+\eta_{n}}\right), \quad n \geq 1 .
\end{aligned}
$$

Taking now an arbitrary $\eta>0$ and $n$ enough large so that $-\eta<\left(t \eta_{n}+e_{n}\right) /(1+$ $\left.\eta_{n}\right)<\eta$, for fixed $t$, we obtain

$$
G_{n, Z}(t-\eta) \leq G_{n, Y}(t) \leq G_{n, Z}(t+\eta) .
$$

Letting $n \rightarrow \infty$ in the above inequalities, from (3.22) we find that

$$
e^{-e^{-(t-\eta)}} \leq \lim \inf _{n \rightarrow \infty} G_{n, Y}(t) \leq \lim \sup _{n \rightarrow \infty} G_{n, Y}(t) \leq e^{-e^{-(t+\eta)}}
$$

for all $\eta>0$. Letting now $\eta \rightarrow 0^{+}$, the required result stated in (1.10) follows from the continuity of the distribution function $e^{-e^{-t}}$. 


\section{Appendix}

Proof of (3.12). First, we recall eq. (2.40) of Lemma 8. Our goal is to represent the $m$ th partial sum $D_{m}(s)$, defined by (2.39), using the inversion formula given by Thm. 3.1 in the Supplement of [18] (see also formula (3.4) there). Instead of $D(s)$, we shall consider now the Dirichelet series $\tilde{D}(s)=D(s+\rho-1)$ (see (2.19) and condition $\left(M_{1}\right)$ ). It converges absolutely for $\Re(s)=\sigma>1$. Lemma 6 implies that the coefficients of $D(s+\rho-1)$ satisfy

$$
b_{k} k^{-\rho+1}=o\left(k^{\rho}\right) k^{-\rho+1}=o(k)<\tilde{c} k,
$$

for some constant $\tilde{c}>0$ and all $k$ (in other words, $\Phi(x)=x$ in Thm. 3.1 of [18; Supplement]). Furthermore, from condition $\left(M_{1}\right)$ it follows that

$$
\sum_{k=1}^{\infty} b_{k} k^{-\rho+1} k^{-\sigma}=\frac{A}{\sigma-1}+\phi(s),
$$

where $\phi(s)$ denotes a function which is analytic for $\sigma \geq-C_{0}$. Hence

$$
\sum_{k=1}^{\infty} b_{k} k^{-\rho+1} k^{-\sigma}=O\left((\sigma-1)^{-1}\right), \quad \sigma \rightarrow 1^{+} .
$$

So, the conditions of Thm. 3.1 (the Supplement of [18]) are satisfied and by its second part we conclude that, for large enough $T>0, \Delta>1$ and $d>0$, we have

$$
\begin{aligned}
& D_{m}(w+\rho-1)+\frac{1}{2} b_{m+1}(m+1)^{-\rho+1}(m+1)^{-w} \\
& =\frac{1}{2 \pi i} \int_{d-i T}^{d+i T} D(w+z+\rho-1) \frac{(m+1)^{z}}{z} d z+O\left(\frac{m^{d}}{(\Delta+d) T}\right) \\
& +O\left(\frac{m^{1-\Delta} \log m}{T}\right), \quad w=1+\Delta+i y,-\infty<y<\infty .
\end{aligned}
$$

Lemma 6 implies that the second term in the left-hand side of (A.1) is $o\left(m^{-\Delta}\right)$ as $m \rightarrow \infty$. To compute the integral in the right-hand side of (A.1), we set $d=1 / \log m, m \geq 2$, and use a contour integral around the rectangle $d-i T, d+$ $i T,-C_{0}-\rho-\Delta+i T,-C_{0}-\rho-\Delta-i T$. Using condition $\left(M_{2}\right)$, we estimate the integral over the end segment $\left(-C_{0}-\rho-\Delta+i T,-C_{0}-\rho-\Delta-i T\right)$ by $O\left(T^{C_{1}+1} m^{-C_{0}-\rho-\Delta}\right)$. Hence, it tends to 0 as $m, T \rightarrow \infty$, provided $T=$ $o\left(m^{\left(C_{0}+\rho+\Delta\right) /\left(C_{1}+1\right)}\right)$. The integrals on the segments $\left(-C_{0}-\rho-\Delta+i T, d+i T\right)$ and $\left(-C_{0}-\rho-\Delta-i T, d-i T\right)$ are easily estimated. By condition $\left(M_{2}\right)$ and the choice of $d$ both are of order

$$
O\left(T^{C_{1}-1} \int_{-C_{0}-\rho-\Delta}^{1 / \log m}(m+1)^{\sigma} d \sigma\right)=O\left(\frac{T^{C_{1}-1} m^{1 / \log m}}{\log m}\right)=O\left(\frac{T^{C_{1}-1}}{\log m}\right)
$$


Therefore, we conclude that $T$ should satisfy

$$
T=\left\{\begin{array}{lll}
o\left(m^{\left(C_{0}+\rho+\Delta\right) /\left(C_{1}+1\right)}\right) & \text { if } & C_{1} \leq 1 \\
o\left((\log m)^{1 /\left(C_{1}-1\right)}\right) & \text { if } & C_{1}>1
\end{array}\right.
$$

Further on we shall assume that $T=T(m) \rightarrow \infty$ as $m \rightarrow \infty$ and $m$ and $T$ satisfy (A.2), where the constants $C_{0}, \rho$ and $C_{1}$ are defined by conditions $\left(M_{1}\right)$ and $\left(M_{2}\right)$ and $\Delta>1$ is fixed. Thus, all integrals on the end segments except the integral on $(d-i T, d+i T)$ are close to 0 for enough large $m$ and $T$. The same obviously holds for both $O$-estimates in the right-hand side of (A.1). So, we can compute $D_{m}(w+\rho-1)$ summing up the residues of the integrand in (A.1).

Inside the contour of integration it has only two simple poles: at $z=1-w$ and $z=0$. Thus, we obtain

$$
\begin{aligned}
& D_{m}(w+\rho-1)=\frac{A(m+1)^{1-w}}{1-w}+D(w+\rho-1)+\Omega_{m}, \\
& w=1+\Delta+i y,-\infty<y<\infty,
\end{aligned}
$$

where $\Omega_{m}$ equals the sum of all negligible terms described above. Clearly,

$$
\Omega_{m} \rightarrow 0, \quad m \rightarrow \infty
$$

for $m$ and $T$ satisfying (A.2). Setting in (A.3) $w=s-\rho+1$ and substituting this expression into (2.40), we arrive at (3.12).

Proof of (3.13). (3.12) and (2.41) imply that

$$
\begin{aligned}
& F_{m, b}\left(e^{-\alpha_{n}}\right)=\frac{A}{2 \pi i} \int_{\rho+\Delta-i \infty}^{\rho+\Delta+i \infty} \alpha_{n}^{-s} \Gamma(s) \zeta(s+1) \frac{(m+1)^{\rho-s}}{\rho-s} d s \\
& +F_{b}\left(e^{-\alpha_{n}}\right)+\frac{A \Omega_{m}}{2 \pi i} \int_{\rho+\Delta-i \infty}^{\rho+\Delta+i \infty} \alpha_{n}^{-s} \Gamma(s) \zeta(s+1) d s .
\end{aligned}
$$

The last integral represents an inverse Mellin transform whose original (see (3.16) and [7; Appendix B.7]) is $H_{2}\left(\alpha_{n}\right)=-\log \left(1-e^{-\alpha_{n}}\right)=O\left(-\log \alpha_{n}\right)$, as $n \rightarrow \infty$. Hence assumption (2.28) and (A.4) imply that

$$
\begin{aligned}
& F_{m, b}\left(e^{-\alpha_{n}}\right)=\frac{A}{2 \pi i} \int_{\rho+\Delta-i \infty}^{\rho+\Delta+i \infty} \alpha_{n}^{-s} \Gamma(s) \zeta(s+1) \frac{(m+1)^{\rho-s}}{\rho-s} d s \\
& +F_{b}\left(e^{-\alpha_{n}}\right)+o\left(-\log \alpha_{n}\right) .
\end{aligned}
$$

To obtain (3.13) it is enough to replace this expression into (3.11), change the variable $s$ in the above integral by $s+\rho$ and observe that $-\log \alpha_{n}=o\left(\alpha_{n}^{-\rho}\right)$.

\section{Acknowledgements}

The author would like to thank the referee for his valuable comments and especially for indicating some defects contained in previous versions of the paper. 


\section{References}

[1] M. Abramovitz and I.A. Stegun, Handbook of Mathemathical Functions with Formulas, Graphs and Mathematical Tables, Dover Publ., Inc. (New York, 1965).

[2] G.E. Andrews, The Theory of Partitions, Encyclopedia Math. Appl. 2, Addison-Wesley (Reading, MA, 1976).

[3] R. Arratia, A.D. Barbour and S. Tavaré, Logarithmic Combinatorial Structures: a Probabilistic Approach, Europ. Math. Soc. (Zürich, 2003).

[4] F.C. Auluck, S. Chowla and H. Gupta, On the maximum value of the number of partitions of $n$ into $k$ parts, J. Indian Math. Soc., 6 (1942), 105-112.

[5] H. Bateman and A. Erdélyi, Tables of Integral Transforms, McGraw-Hill (New York, 1954).

[6] P. Erdős and J. Lehner, The distribution of the number of summands in the partition of a positive integer, Duke Math.J., 8 (1941), 335-345.

[7] Ph. Flajolet and R. Sedgewick, Analytic Combinatorics, Cambridge Univ. Press (Cambridge, 2009).

[8] G.A. Freiman and B.L. Granovsky, Asymptotic formula for a partition function of reversable coagulation-fragmentation processes, Israel J. Math., 130 (2002), 259-279.

[9] B. Fristedt, The structure of random partitions of large integers, Trans. Amer. Math. Soc., 337 (1993), 703-735.

[10] B. Granovsky, D. Stark and M. Erlihson, Meinardus theorem on weighted partitions: Extensions and a probabilistic proof, Adv. Appl. Math., 41 (2008), 307-328.

[11] W.K. Hayman, A generalization of Stirling's formula, J. Reine Angew. Math., 196 (1956), 67-95.

[12] J. Korevaar, Tauberian Theory, Springer (Berlin, 2004).

[13] G. Meinardus, Asymptotische Aussagen über Partitionen, Math. Z., 59 (1954), 388-398.

[14] L. Mutafchiev, The size of the largest part of random plane partitions of large integers, Integers: Electron. J. Comb. Number Theory, 6 (2006), \#A13.

[15] L. Mutafchiev, Limit theorems for the number of parts in a random weighted partition, Electron. J. Comb., 18(1) (2011), \#P206. 
[16] A. Nijenhuis and H. Wilf, Combinatorial Algorithms, 2nd Ed., Academic Press (New York, 1978).

[17] B. Pittel, On dimensions of a random solid diagram, Combinatorics Probab. Comput., 14 (2005), 873-895.

[18] K. Prachar, Primzahlverteilung, Springer (Berlin, 1957).

[19] R.P. Stanley, Enumerative Combinatorics 2, Vol. 62 of Cambridge Studies in Advanced Mathematics, Cambridge Univ. Press (Cambridge, 1999).

[20] E.C. Titchmarsh, The Theory of Functions, Oxford Univ. Press (Oxford, 1939).

[21] A. Vershik, Statistical mechanics of combinatorial partitions and their limit configurations, Funct. Anal. Appl., 30 (1996), 90-105.

[22] A. Vershik and Yu. Yakubovich, Fluctuations of the maximal particle energy of the quantum ideal gas and random partitions, Comm. Math. Phys., 261 (2006), 759-769.

[23] E.T. Whittaker and G.N. Watson, A Course of Modern Analysis, Cambridge Univ. Press (Cambridge, 1927).

[24] H. Wilf, generatingfunctionology, Academic Press (New York, 1994). 